UT-Komaba 98-5

Feburary 1998

\title{
Determinant formula for the six-vertex model with reflecting end
}

\author{
Osamu Tsuchiya 1 \\ Institute of Physics, University of Tokyo, \\ Komaba, Meguro-ku, Tokyo 153, Japan
}

\begin{abstract}
Using the Quantum Inverse Scattering Method for the XXZ model with open boundary conditions, we obtained the determinant formula for the six vertex model with reflecting end.
\end{abstract}

\footnotetext{
${ }^{1}$ E-mail address: otutiya@hep1.c.u-tokyo.ac.jp
} 


\section{Introducntion}

Determinant representations of correlation functions of one dimensional quantum integrable models were studied extensively[1]. Especially Essler et. al. studied the correlation functions of XXZ model with periodic boundary conditions [四]. In the calculation of scalar products of the XXZ model, the relation between the scalar products of the model and the partition function of the six-vertex with domain wall boundary conditions is essential [2]. Izergin et. al. used this relation to obtain the determinant formula for the partition function of the six-vertex model with domain wall boundary conditions [5].

On the other hand, integrable models with open boundary conditions are interesting. Integrable model with open boundary conditions are related to the $B_{N}$ type Weyl group [6] and in condenced matter physics they are related to the impurity problems in the Luttinger liquid [7].

Correlation function of the $1+1$ dimensional delta function interacting Bose gas with open boundary conditions were studied in [8].

For the XXZ model with open boundary conditions, the algebraic Bethe ansatz of the model can be used to calculate the partition function of the six-vertex model with reflecting boundary condition at one boundary and domain wall boundary conditions at other three boundaries. (In this paper we call six vertex model with this type of boundary conditions six vertex model with reflecting end.) In this paper, we obtain the determinant formula for the above six-vertex model using above relation.

\section{Quantum Inverse Scattering Method for the Open XXZ Model}

Let us start with a brief review of the Quantum Inverse Scattering Method for the XXZ model with open boundary conditions [9]. The trigonometrical solution of the Yang-Baxter equation is given by

$$
\begin{aligned}
R_{00^{\prime}}(\lambda)= & \frac{1}{2}\left\{\left[\frac{\sinh (\lambda-2 i \eta)-\sinh (2 i \eta)}{\sinh (\lambda)}\right] 1_{0} \otimes 1_{0^{\prime}}+\sigma_{0}^{x} \otimes \sigma_{0^{\prime}}^{x}+\sigma_{0}^{y} \otimes \sigma_{0^{\prime}}^{y}\right. \\
& \left.+\left[\frac{\sinh (\lambda-2 i \eta)+\sinh (2 i \eta)}{\sinh (\lambda)}\right] \sigma_{0}^{z} \otimes \sigma_{0^{\prime}}^{z}\right\}
\end{aligned}
$$

where $R_{00^{\prime}}(\mu)$ acts as a linear operator on the tensor product of two auxiliary spaces. The $L$ operators are given by $L_{n}(\lambda)=\sinh (\lambda) R_{0 n}(\lambda) P_{0 n}$ where 0 denotes the auxiliary space, $n$ denote the $n$-th quantum space and 


$$
P_{0 n}=(1 / 2)\left(1_{0} \otimes 1_{n}+\sigma_{0}^{x} \otimes \sigma_{n}^{x}+\sigma_{0}^{y} \otimes \sigma_{n}^{y}+\sigma_{0}^{z} \otimes \sigma_{n}^{z}\right) \text { is a permutation operator. }
$$

Thus

$$
\begin{aligned}
L_{n}(\lambda)= & \frac{1}{2}\left\{[\sinh (\lambda-2 i \eta)+\sinh (\lambda)] 1_{0} \otimes 1_{n}-\sinh (2 i \eta)\left[\sigma_{0}^{x} \otimes \sigma_{n}^{x}+\sigma_{0}^{y} \otimes \sigma_{n}^{y}\right]\right. \\
& \left.+[\sinh (\lambda-2 i \eta)-\sinh (\lambda)] \sigma_{0}^{z} \otimes \sigma_{n}^{z}\right\}
\end{aligned}
$$

As a consequence of the Yang-Baxter equation, the $L$ operators satisfy the fundamental commutation relations

$$
R(\lambda-\mu)\left(L_{n}(\lambda) \otimes L_{n}(\mu)\right)=\left(L_{n}(\mu) \otimes L_{n}(\lambda)\right) R(\lambda-\mu) .
$$

The monodromy matrix of inhomogeneous XXZ model is constructed from the $L$ operators as:

$$
T(\lambda, \boldsymbol{\omega})=L_{L}\left(\lambda-\omega_{L}\right) L_{L-1}\left(\lambda-\omega_{L-1}\right) \cdots L_{1}\left(\lambda-\omega_{1}\right)
$$

This matrix satisfies fundamental commutation relations similar to (4)

$$
R(\lambda-\mu)(T(\lambda, \boldsymbol{\omega}) \otimes T(\mu, \boldsymbol{\omega}))=(T(\mu, \boldsymbol{\omega}) \otimes T(\lambda, \boldsymbol{\omega}) R(\lambda-\mu) .
$$

For the model with open boundary conditions, in addition to the Yang-Baxter equation, the reflection equation [9] plays an important role,

$$
R_{12}(\lambda-\mu) K_{1}(\lambda) R_{12}(\lambda+\mu) K_{1}(\mu)=K_{1}(\mu) R_{12}(\lambda+\mu) K_{1}(\lambda) R_{12}(\lambda-\mu),
$$

where $K_{j}(\lambda)$ is the $2 \times 2$ matrix which act to the $j$-th auxiliary space and is related to the reflection of the particle with rapidity $\lambda$ at the boundaries. In the present paper, we concentrate to the diagonal solution of the reflection equation

$$
K(\lambda, \xi)=\frac{1}{\sinh (\xi)}\left(\begin{array}{cc}
\sinh (\xi+\lambda) & 0 \\
0 & \sinh (\xi-\lambda)
\end{array}\right) .
$$

For the model with open boundary conditions, Sklyanin's monodromy matrix

$$
U(\lambda, \boldsymbol{\omega})=T(\lambda, \boldsymbol{\omega}) K\left(\lambda, \xi_{-}\right) \tilde{T}(\lambda, \boldsymbol{\omega})=\left(\begin{array}{ll}
\mathcal{A}(\lambda, \boldsymbol{\omega}) & \mathcal{B}(\lambda, \boldsymbol{\omega}) \\
\mathcal{C}(\lambda, \boldsymbol{\omega}) & \mathcal{D}(\lambda, \boldsymbol{\omega})
\end{array}\right)
$$

where

$$
\begin{aligned}
& \tilde{T}(\lambda, \boldsymbol{\omega})=L_{1}\left(\lambda+\omega_{1}\right) L_{2}\left(\lambda+\omega_{2}\right) \cdots L_{L}\left(\lambda+\omega_{L}\right) \\
& \propto T^{-1}(-\lambda, \boldsymbol{\omega})
\end{aligned}
$$

is fundamental, where $\mathcal{A}(\lambda, \boldsymbol{\omega}), \mathcal{B}(\lambda, \boldsymbol{\omega}), \mathcal{C}(\lambda, \boldsymbol{\omega}), \mathcal{D}(\lambda, \boldsymbol{\omega})$ are operators which act on the quantum spaces. 
$U(\lambda, \boldsymbol{\omega})$ also satisfies reflection equation

$$
\begin{aligned}
& R_{12}(\lambda-\mu) U_{1}(\lambda, \boldsymbol{\omega}) R_{12}(\lambda+\mu) U_{1}(\mu, \boldsymbol{\omega}) \\
& =U_{1}(\mu, \boldsymbol{\omega}) R_{12}(\lambda+\mu) U_{1}(\lambda, \boldsymbol{\omega}) R_{12}(\lambda-\mu)
\end{aligned}
$$

The reflection equation is rewritten as the commutation relations of the matrix elements $\mathcal{A}, \mathcal{B}, \mathcal{C}, \mathcal{D}$

$$
\begin{aligned}
& {[\mathcal{A}(\lambda), \mathcal{A}(\mu)]=-[\mathcal{D}(\lambda), \mathcal{D}(\mu)]} \\
& =\frac{\sinh (2 i \eta)}{\sinh (\lambda+\mu-2 i \eta)}\{\mathcal{B}(\lambda) \mathcal{C}(\mu)-\mathcal{B}(\mu) \mathcal{C}(\lambda)\} \\
& =-\frac{\sinh (2 i \eta)}{\sinh (\lambda+\mu-2 i \eta)}\{\mathcal{C}(\lambda) \mathcal{B}(\mu)-\mathcal{C}(\mu) \mathcal{B}(\lambda)\} \\
& =\frac{\sinh (\lambda-\mu) \sinh (\lambda+\mu)}{\sinh ^{2}(2 i \eta)-\sinh (\lambda+\mu-2 i \eta)}[\mathcal{A}(\lambda), \mathcal{D}(\mu)] \\
& {[\mathcal{B}(\lambda), \mathcal{B}(\mu)]=[\mathcal{C}(\lambda), \mathcal{C}(\mu)]=0} \\
& {[\mathcal{B}(\lambda), \mathcal{C}(\mu)]=\frac{\sinh (2 i \eta)}{\sinh (\lambda-\mu) \sinh (\lambda+\mu-2 i \eta)} \times} \\
& \times\{\sinh (\lambda+\mu)[\mathcal{D}(\lambda) \mathcal{A}(\mu)-\mathcal{D}(\mu) \mathcal{A}(\lambda)] \\
& \quad+\sinh (\lambda-\mu)[\mathcal{A}(\lambda) \mathcal{A}(\mu)-\mathcal{D}(\mu) \mathcal{D}(\lambda)]\}
\end{aligned}
$$

From the above commutation relations (13) Sklyanin's transfer matrices

$$
\begin{aligned}
& \tau(\lambda)=\operatorname{tr}_{0}(K(-\lambda+2 i \eta) U(\lambda)) \\
& =\left(1 / \sinh \left(\xi_{+}\right)\right)\left[\sinh \left(\xi_{+}-\lambda+2 i \eta\right) \mathcal{A}(\lambda)+\sinh \left(\xi_{+}+\lambda-2 i \eta\right) \mathcal{D}(\lambda)\right]
\end{aligned}
$$

are commutative for any values of spectral parameter $[\tau(\lambda), \tau(\mu)]$.

Consequently, $\tau(\lambda)$ generate the infinite number of conserved quantities. For example the Hamiltonian is given by

$$
\begin{aligned}
& \left.\frac{d}{d \lambda} \tau(\lambda)\right|_{\lambda=0} \\
& =-\frac{1}{\sinh (2 i \eta)} \sum_{n=1}^{L-1}\left\{\sigma_{n}^{x} \sigma_{n+1}^{x}+\sigma_{n}^{y} \sigma_{n+1}^{y}+\cosh (2 i \eta) \sigma_{n}^{z} \sigma_{n+1}^{z}\right\} \\
& +\operatorname{coth}\left(\xi_{-}\right) \sigma_{1}^{z}-\operatorname{coth}\left(\xi_{+}\right) \sigma_{L}^{z}+\text { constant }
\end{aligned}
$$

In the algebraic Bethe ansatz, we construct the simultaneous eigenstates of the conserved quantities by applying the some elements (creation operator) of the monodromy matrix to the pseudovacuum. We choose as a pseudovacuum the state whose spins are all up

$$
|0\rangle=\otimes_{j=1}^{L}|+\rangle_{j}
$$


This pseudovacuum is the eigenstate of $\mathcal{A}$ and $\mathcal{D}$ and is annihilated by $\mathcal{C}$

$$
\begin{aligned}
& \mathcal{A}(\lambda)|0\rangle=a(\lambda)|0\rangle, \\
& \mathcal{D}(\lambda)|0\rangle=d(\lambda)|0\rangle, \\
& \mathcal{C}(\lambda)|0\rangle=0,
\end{aligned}
$$

where

$$
\begin{aligned}
& a(\lambda)=\sinh \left(\xi_{-}+\lambda\right) \prod_{n=1}^{L}\left\{\sinh \left(\lambda-\omega_{n}-2 i \eta\right) \sinh \left(\lambda+\omega_{n}-2 i \eta\right)\right\}, \\
& d(\lambda)=\sinh \left(\xi_{-}-\lambda\right) \prod_{n=1}^{L}\left\{\sinh \left(\lambda-\omega_{n}\right) \sinh \left(\lambda+\omega_{n}\right)\right\} .
\end{aligned}
$$

In other words $\mathcal{B}$ has the role of the creation operator and the eigenstates of the Hamiltonian are of the form $\prod_{n=1}^{N} \mathcal{B}\left(\lambda_{n}, \boldsymbol{\omega}\right)|0\rangle$ if rapidities $\lambda_{n}$ satisfy the Bethe ansatz equations.

In the algebraic Bethe ansatz eigenstates and dual states of eigenstates are respectively given by

$$
\mathcal{B}\left(\lambda_{L}\right) \cdots \mathcal{B}\left(\lambda_{1}\right)|0\rangle
$$

and

$$
\langle 0| \mathcal{C}\left(\mu_{1}\right) \cdots \mathcal{C}\left(\mu_{L}\right)
$$

\section{Inhomogeneous six-vertex model with reflect- ing end}

For the scalar products between the eigenstates the following relation holds

$$
\begin{aligned}
& \left\langle 0\left|\mathcal{C}\left(\mu_{1}\right) \cdots \mathcal{C}\left(\mu_{L}\right) \mathcal{B}\left(\lambda_{L}\right) \cdots \mathcal{B}\left(\lambda_{1}\right)\right| 0\right\rangle \\
& =\left\langle 0\left|\mathcal{C}\left(\mu_{1}\right) \cdots \mathcal{C}\left(\mu_{L}\right)\right| \overline{0}\right\rangle\left\langle\overline{0}\left|\mathcal{B}\left(\lambda_{L}\right) \cdots \mathcal{B}\left(\lambda_{1}\right)\right| 0\right\rangle
\end{aligned}
$$

where $|\overline{0}\rangle$ is the quantum state with all spins are down $\prod_{n=1}^{L}|-\rangle$. Note that

$$
Z_{L}(\{\lambda\},\{\omega\})=\left\langle\overline{0}\left|\mathcal{B}\left(\lambda_{L}\right) \cdots \mathcal{B}\left(\lambda_{1}\right)\right| 0\right\rangle,
$$

is nothing but the partition function of the inhomogeneous 6 vertex model on a $L \times 2 L$ lattice with domain wall boundary condition for the lower, upper and left boundaries and reflecting end for the right boundary (see Fig. 1.).

$$
\left.\left.\mathcal{B}\left(\lambda_{n}\right)={ }_{n}^{0}(+|U(\lambda, \omega)|-)_{n}^{0} \text { where } \mid+\right)_{n}^{0} \text { and } \mid-\right)_{n}^{0}
$$


are the $n$-th auxiliary states with spins + and respectively $-\left({ }_{n}^{0}\left(+\mid\right.\right.$ and ${ }_{n}^{0}(-\mid$ are dual states with spins + and respectively -$)$. Here we assign the auxiliary space to each $\mathcal{B}\left(\lambda_{n}\right)$.

Now we derive the recursion relations to the partition function $Z_{L}(\{\lambda\},\{\omega\})$.

If the inhomogenuity $\omega_{L}$ is choosen to be $\omega_{L}=-\lambda_{1}$ then the partition function of the $L \times 2 L$ lattice is related to the partiton function of the $L-1 \times 2(L-1)$ lattice.

The $L$ operators are regular i.e.

$$
L_{L}(0)=-\sinh (2 i \eta) P_{o L}
$$

Thus

$$
\begin{aligned}
& \mathcal{B}\left(\lambda_{1}\right) \prod_{j=1}^{L}\left|+>_{j}\right|_{\omega_{L}=-\lambda_{1}} \\
& =-\sinh (2 i \eta)_{1}^{o}\left(+\left|L_{L}\left(\lambda_{1}-\omega_{L}\right) \cdots L_{L-1}\left(\lambda_{1}+\omega_{L-1}\right) P_{o L}\right|-\right)_{1}^{o} \prod_{j=1}^{L}|+\rangle_{j} \\
& =-\sinh (2 i \eta)_{1}^{o}\left(+\left|L_{L}\left(\lambda_{1}-\omega_{L}\right) \cdots L_{L-1}\left(\lambda_{1}+\omega_{L-1}\right)\right|+\right)_{1}^{o}|-\rangle_{L} \prod_{j=1}^{L-1}|+\rangle_{j} .
\end{aligned}
$$

Noting that $\mid+)_{1}|+\rangle_{n}$ is the eigenstate of $L_{n}\left(\lambda_{1}\right)$

$$
\begin{gathered}
\left.\left.L_{n}\left(\lambda_{1}\right) \mid+\right)_{1}|+\rangle_{n}=\sinh \left(\lambda_{1}-2 i \eta\right) \mid+\right)_{1}|+\rangle_{n}, \\
\left.\left.K\left(\lambda_{1}, \xi_{-}\right) \mid+\right)_{1}=\frac{\sinh \left(\xi_{-}+\lambda_{1}\right)}{\sinh \left(\xi_{-}\right)} \mid+\right)_{1}
\end{gathered}
$$

and

$$
{ }_{1}\left(+\left|L_{L}\left(\lambda_{n}-\omega_{L}\right)\right|-\right\rangle_{L}=2 \sinh \left(\lambda_{n}-\omega_{L}\right)_{1}(+||-\rangle_{L}
$$

We obtain

$$
\begin{aligned}
& \mathcal{B}\left(\lambda_{1}\right) \prod_{j=1}^{L}\left|+>_{j}\right|_{\omega_{L}=-\lambda_{1}} \\
& =-\frac{\sinh \left(\xi_{1}+\lambda_{1}\right)}{\sinh \left(\xi_{-}\right)} \sinh (2 i \eta) \sinh \left(2 \lambda_{1}\right) \\
& \times \prod_{j=1}^{L-1} \sinh \left(\lambda_{1}-\omega_{j}-2 i \eta\right) \sinh \left(\lambda_{1}+\omega_{j}-2 i \eta\right) \\
& \times|-\rangle_{L} \prod_{j=1}^{L-1}|+\rangle_{j},
\end{aligned}
$$


Using

$$
\left.\left.L_{L}\left(\lambda_{n}+\omega_{L}\right) \mid-\right)_{n}|-\rangle_{L}=\sinh \left(\lambda_{n}+\omega_{L}-2 i \eta\right) \mid-\right)_{n}|-\rangle_{L}
$$

together with equation (30) and the locality of the $L$ operators we find the relation

$$
\mathcal{B}\left(\lambda_{n}\right)|-\rangle_{L}=\sinh \left(\lambda_{n}+\omega_{L}-2 i \eta\right) \sinh \left(\lambda_{n}-\omega_{L}\right)|-\rangle_{L} \mathcal{B}_{L-1}\left(\lambda_{n}\right)
$$

where

$$
\begin{aligned}
\mathcal{B}_{L-1}\left(\lambda_{n}\right)= & n\left(+\mid L_{L-1}\left(\lambda_{n}-\omega_{L-1}\right) \cdots L_{1}\left(\lambda_{n}-\omega_{1}\right) K\left(\lambda_{n}, \xi_{-}\right)\right. \\
& \left.\times L_{1}\left(\lambda_{n}+\omega_{1}\right) \cdots L_{L-1}\left(\lambda_{n}+\omega_{L-1}\right) \mid-\right)_{n} .
\end{aligned}
$$

lemma 1-a

The partition function of the model on the $L \times 2 L$ lattice is related to hte one on $L-1 \times 2(L-1)$ lattice

$$
\begin{aligned}
& \left.Z_{L}(\{\lambda\},\{\omega\})\right|_{\omega_{L}=-\lambda_{1}} \\
& =-\frac{\sinh \left(\xi_{-}+\lambda_{L}\right)}{\sinh \left(\xi_{-}\right)} \sinh (2 i \eta) \sinh \left(2 \lambda_{L}\right) \\
& \times \prod_{j=1}^{L-1} \sinh \left(\lambda_{L}-\omega_{j}-2 i \eta\right) \sinh \left(\lambda_{L}+\omega_{j}-2 i \eta\right) \\
& \times \prod_{n=1}^{L-1} \sinh \left(\lambda_{n}+\omega_{L}-2 i \eta\right) \sinh \left(\lambda_{n}-\omega_{L}\right) Z_{L-1}(\{\lambda\},\{\omega\}) .
\end{aligned}
$$

Here $Z_{M}(\{\lambda\},\{\omega\})$ is the partition function for the model on $M \times 2 M$ lattice

$$
Z_{M}(\{\lambda\},\{\omega\})=\prod_{j=1}^{M}{ }_{j}\left\langle-\left|\prod_{n=1}^{M} \mathcal{B}_{M}\left(\lambda_{n}, \boldsymbol{\omega}\right) \prod_{j=1}^{M}\right|+\right\rangle_{j},
$$

which depend on $\lambda_{1} \sim \lambda_{M}$ and $\omega_{1} \sim \omega_{M}$.

lemma 1-b

Folowing the same line of argument as above we have other type of recursion relations 


$$
\begin{aligned}
& \left.Z_{L}(\{\lambda\},\{\omega\})\right|_{\omega_{L}=\lambda_{1}} \\
& =-\frac{\sinh \left(\xi_{-}-\lambda_{L}\right)}{\sinh \left(\xi_{-}\right)} \sinh (2 i \eta) \sinh \left(2 \lambda_{L}\right) \\
& \times \prod_{j=1}^{L-1} \sinh \left(\lambda_{L}-\omega_{j}-2 i \eta\right) \sinh \left(\lambda_{L}+\omega_{j}-2 i \eta\right) \\
& \prod_{n=1}^{L-1} \sinh \left(\lambda_{n}-\omega_{L}-2 i \eta\right) \sinh \left(\lambda_{n}+\omega_{L}\right) Z_{L-1}(\{\lambda\},\{\omega\}) .
\end{aligned}
$$

Lemma 2

$$
Z_{1}(\{\lambda\},\{\omega\})={ }_{1}\langle\overline{0}|\mathcal{B}(\lambda)| 0\rangle_{1}
$$

is explicitly calculated as

$$
Z_{1}(\{\lambda\},\{\omega\})=-\frac{\sinh (2 i \eta)}{\sinh \left(\xi_{-}\right)}[\sinh (\xi+\lambda) \sinh (\lambda-\omega)+\sinh (\xi-\lambda) \sinh (\lambda+(\dot{3}) 9 .)
$$

Lemma 3

$\left\langle\overline{0}\left|\mathcal{B}\left(\lambda_{L}\right) \cdots \mathcal{B}\left(\lambda_{1}\right)\right| 0\right\rangle$ depend on each $\lambda_{n}$ as

$$
\begin{aligned}
& \left\langle\overline{0}\left|\mathcal{B}\left(\lambda_{L}\right) \cdots \mathcal{B}\left(\lambda_{1}\right)\right| 0\right\rangle \\
& =e^{-2 L \lambda_{n}} P_{2 L}\left(e^{2 \lambda_{n}}\right)
\end{aligned}
$$

where $P_{2 L}(x)$ is the polynomial of degree $2 L$.

This is proved along the same line of argument as in reference [5].

\section{Determinant formula for the six vertex model with reflecting end}

The lemmas in the last section determine the partition function of the model uniquely. Theorem: The partition function of the six vertex model with reflecting end is given by

$$
\begin{aligned}
& Z_{L}(\{\lambda\},\{\omega\}) \\
& =\prod_{j=1}^{L} \prod_{n=1}^{L} \sinh \left(\lambda_{j}-\omega_{n}-2 i \eta\right) \sinh \left(\lambda_{j}-\omega_{n}\right) \sinh \left(\lambda_{j}+\omega_{n}-2 i \eta\right) \sinh \left(\lambda_{j}+\omega_{n}\right) \\
& \times\left[\prod_{j>k} \sinh \left(\lambda_{j}-\lambda_{k}\right) \sinh \left(\lambda_{j}+\lambda_{k}-2 i \eta\right) \prod_{n>m} \sinh \left(\omega_{n}-\omega_{m}\right) \sinh \left(\omega_{n}+\omega_{m}\right)\right]^{-1} \\
& \times \operatorname{det} M
\end{aligned}
$$


where

$$
\begin{aligned}
& M_{j n}=\frac{\sinh (2 i \eta)}{\sinh (\xi) \sinh \left(\lambda_{j}-\omega_{n}-2 i \eta\right) \sinh \left(\lambda_{j}+\omega_{n}-2 i \eta\right)} \\
& \times\left\{\frac{\sinh \left(\xi_{-}+\lambda_{j}\right)}{\sinh \left(\lambda_{j}+\omega_{n}\right)}+\frac{\sinh \left(\xi_{-}-\lambda_{j}\right)}{\sinh \left(\lambda_{j}-\omega_{n}\right)}\right\}
\end{aligned}
$$

\section{Conclusion}

In this paper the determinant formula for the partition function of the six-vertex model with reflecting end is given. It is the determinant formula corresponding to the Quantum Inverse Scattering Method with open boundary conditions.

In the model with open boundary conditions, as opposed to the model with periodic boundary conditions, one can not determine the scalar product from the six-vertex model with reflecting end. To calculate the scalar products and correlation functions for the open XXZ model is interesting.

\section{References}

[1] V. E. Korepin, N. M. Bogoliubov and A. G. Izergin, Quantum Inverse Scattering Method and Correlation Function.

[2] V. E. Korepin, Commun. Math. Phys. 86 (1982) 391.

[3] F. H. L. Essler, H. Frahm, A. G. Izergin and V. E. Korepin, Comm. Math. Phys. 174 (1995) 191.

[4] F. H. L. Essler, H. Frahm, A. R. Its and V. E. Korepin, Integro-Difference Equation for a correlation function of the spin-1/2 Heisenberg XXZ chain, preprint (1995) cond-mat/9503142.

[5] A. G. Izergin, D. A. Coker and V. E. Korepin, J. Phys. A: Math. Gen. 25 (1992) 4315.

[6] T. Yamamoto and O. Tsuchiya, J. Phys. A: Math.Gen. 29 (1996) 3977.

[7] O. Tsuchiya and T. Yamamoto, J. Phys. Soc. Japan 66 (1997) 1950.

[8] T. Kojima, Dynamical correlation functions for an impenetrable Bose gas with open boundary conditions, preprint (1997) 
[9] E. K. Sklyanin, J. Phys. A: Math. Gen. 21 (1988) 2375. 